\title{
Hybrid Iterated Kalman Particle Filter for Object Tracking Problems
}

\author{
Amr M. Nagy ${ }^{1}$, Ali Ahmed ${ }^{2}$ and Hala H. Zayed ${ }^{1}$ \\ ${ }^{1}$ Faculty of Computers and Informatics, Benha University, Banha ElGadeda, Benha, Egypt \\ ${ }^{2}$ Faculty of Computers and Information, Menofia University, Shubin el Kom, Menufia, Egypt \\ \{amr.nagy,hala.zayed\}@fci.bu.edu.eg,ali.ahmed@ci.menofia.edu.eg
}

Keywords: $\quad$ Kalman Filter, Particle Filter, Nonlinear/Non-Gaussian, Object Tracking.

\begin{abstract}
Particle Filters (PFs), are widely used where the system is non Linear and non Gaussian. Choosing the importance proposal distribution is a key issue for solving nonlinear filtering problems. Practical object tracking problems encourage researchers to design better candidate for proposal distribution in order to gain better performance. In this correspondence, a new algorithm referred to as the hybrid iterated Kalman particle filter (HIKPF) is proposed. The proposed algorithm is developed from unscented Kalman filter (UKF) and iterated extended Kalman filter (IEKF) to generate the proposal distribution, which lead to an efficient use of the latest observations and generates more close approximation of the posterior probability density. Comparing with previously suggested methods(e.g PF, PF-EKF, PF-UKF, PF-IEKF), our proposed method shows a better performance and tracking accuracy. The correctness as well as validity of the algorithm is demonstrated through numerical simulation and experiment results.
\end{abstract}

\section{INTRODUCTION}

The increasing interest in the object tracking is motivated by a huge number of promising applications that can now be tackled in real-time applications. These applications include performance analysis, surveillance, video-indexing, smart interfaces, teleconferencing and video compression and so on.

A variety of tracking algorithms have been proposed and implemented. They can be roughly classified into two categories: deterministic methods and stochastic methods. Deterministic methods typically track the object by performing an iterative search for a similarity between the template image and the current one. The algorithms which utilize the deterministic method are background subtraction ((McIvor, 2000); (LIU et al., 2001)), inter-frame difference ((Lipton et al., 1998);(Collins et al., 2000)), optical flow (Meyer et al., 1998), skin color extraction ((kyung-min Cho et al., 2001); (Phung et al., 2003)) and so on. On the other hand, the stochastic methods use the state space to model the underlying dynamics of the tracking system such as Kalman filter (Broida and Chellappa, 1986) and particle filter ((Isard and Blake, 1998); (Ristic et al., 2004); (Sugandi et al., 2009); (Fen and Ming., 2010); (Zhiqiang et al., 2011); (Zhonga et al., 2012) ).

Probabilistic methods have become popular among many researchers. The Kalman filter is a common approach for dealing with target tracking in a probabilistic framework, but it cannot resolve a tracking problem where the model is nonlinear and nonGaussian. The extended Kalman filter can deal with this problem, but still has a problem when the nonlinearity and non-Gaussian cannot be approximated accurately.

Recently, the particle filter method, a numerical method that allows finding an approximate solution to the sequential estimation has proven very successful for nonlinear and non-Gaussian estimation problems. It approximates a posterior probability density of the state such as the object position by using samples which are called particles. A key issue in particle filtering is the selection of the proposal distribution function. In general, it is hard to design such proposals. Now many proposed distributions have been proposed in the literature. For example, the prior, the EKF Gaussian approximation and the UKF proposal are used as the proposal distribution for particle filter ((Gordon et al., 1993); (Arulampalam et al., 2002); (R Van der Merwe, 2000)).

In this paper, a new proposal distribution generating scheme for the particle filtering framework is proposed. The algorithm obtained is named as the hybrid Iterated Kalman particle filter (HIKPF). This algorithm uses hybrid Kalman filter (HKF) to generate 
proposal distribution. In this algorithm, each particle is updated by the UKF and the IEKF sequentially. Through this procedure, efficient use of the latest observations is made, which consequently improves the performance of particle filters.

\section{PARTICLE FILTER}

Considering the following nonlinear system (Arulampalam et al., 2002):

$$
\begin{gathered}
x_{k}=f_{k}\left(x_{k-1}, v_{k-1}\right) \\
y_{k}=h_{k}\left(x_{k}, u_{k}\right)
\end{gathered}
$$

Where $x_{k}$ denotes the system state, and $y_{k}$ denotes the observation at time $k$. The functions $f(\cdot)$ and $h(\cdot)$ represent the system transition model and the measurement model respectively. The process noise $v_{k}$ and the measurement noise $u_{k}$ are assumed independent with known distributions. The prior knowledge of the initial state is given by the probability distribution $P\left(x_{0}\right)$.

\subsection{Recursive Bayesian Estimation}

The objective of the recursive Bayesian state estimation problem is to find the mean and variance of a random variable $x_{k}$ using the conditional probability density function $P\left(x_{k} \mid y_{k}\right)$, using Bayes' formula under following assumptions (Arulampalam et al., 2002):

- The states follow a first-order Markov process.

- The observation are conditionally independent given the state variables.

$Y_{k}$ denotes the set of all the available measurements, i.e The posterior density $P\left(x_{k} \mid y_{k}\right)$ is estimated in two steps: (a) Prediction step, which is computed before obtaining an observation.

$$
P\left(x_{k} \mid y_{k-1}\right)=\int P\left(x_{k} \mid x_{k-1}\right) P\left(x_{k-1} \mid y_{k-1}\right) d x_{k-1}
$$

(b) Update step, which is computed after obtaining an observation

$$
P\left(x_{k} \mid y_{k}\right)=\frac{P\left(y_{k} \mid x_{k}\right) P\left(x_{k} \mid y_{k-1}\right)}{P\left(y_{k} \mid y_{k-1}\right)}
$$

where

$$
P\left(y_{k} \mid y_{k-1}\right)=\int P\left(y_{k} \mid x_{k}\right) P\left(x_{k} \mid y_{k-1}\right) d x_{k}
$$

By substituting Eqs. (3) and (5) in Eq. (4) we can get obtain the final equation:

$$
P\left(x_{k} \mid y_{k}\right)=\frac{P\left(y_{k} \mid x_{k}\right) \int P\left(x_{k} \mid x_{k-1}\right) P\left(x_{k-1} \mid y_{k-1}\right) d x_{k-1}}{\int P\left(y_{k} \mid x_{k}\right) P\left(x_{k} \mid y_{k-1}\right) d x_{k}}
$$

The prediction and update strategy provides an optimal solution to the state estimation problem,which, unfortunately, involves high-dimensional integration. The solution is extremely general and aspects such as multimodality, asymmetries and discontinuities can be incorporated.

\subsection{Solution through Monte Carlo Sampling}

The exact analytical solution to the recursive propagation of the posterior density is difficult to obtain for a general nonlinear system, because it involves high-dimensional integration of unknown density functions (refer to Eqs. (3) and (6)). However, when the process model is linear and noise sequences are zero mean Gaussian white noise sequences, the Kalman filter describes the optimal recursive solution to the sequential state estimation problem (Soderstorm, 2002) While dealing with nonlinear systems, it becomes necessary to develop approximate and computationally tractable sub-optimal solutions to the above sequential Bayesian estimation problem. The particle filter is a numerical method for implementing an optimal recursive Bayesian filter through Monte Carlo simulation. Classical particle filters approximate the distribution $P\left(x_{k} \mid y_{k}\right)$ , using a set of random samples $x_{k}^{i}: i=1, \cdots, N$ together with associated weights $\omega_{k}^{i}: i=1, \cdots, N$ and $x_{k}=x_{j}, j=0, \cdots, k$ is the set of all states up to time $k$. The weights are normalised such that $\sum_{i} \omega_{k}^{i}=1$. Then, the posterior density at $k$ can be approximated as

$$
P\left(x_{k} \mid y_{k}\right) \approx \sum_{i=1}^{N} \omega_{k}^{i} \delta\left(x_{k}-x_{k}^{i}\right)
$$

where $\delta\left(x_{k}-x_{k}^{i}\right)$ denotes the Dirac delta function. The weights $\omega_{k}^{i}$ can be viewed as approximations to the relative posterior probabilities of the particles. It should be noted that the posterior density $P\left(x_{k} \mid y_{k}\right)$ is seldom known. Therefore, it is not possible to draw samples from this distribution. For this reason, $q\left[\left(x_{k}^{i} \mid x_{k-1}^{i}\right), y_{k}\right]$, a proposal density or importance density, is used. At each sampling instant, a sample is drawn from the proposal distribution generated around each particle. To compensate for the difference between the proposal density and the true posterior density, the weights are then computed as follows:

$$
\tilde{\omega}_{k}^{i}=\frac{P\left(x_{k}^{i} \mid y_{k}\right)}{q\left(x_{k}^{i} \mid y_{k}\right)}
$$


and the updated weight equation is:

$$
\tilde{\omega}_{k}^{i}=\frac{P\left(y_{k} \mid x_{k}^{i}\right) P\left(x_{k}^{i} \mid x_{k-1}^{i}\right)}{q\left(x_{k}^{i} \mid x_{k-1}^{i}, y_{k}\right)} \tilde{\omega}_{k-1}^{i}
$$

as a result the normalized weight is given by:

$$
\omega_{k}^{i}=\frac{\tilde{\omega}_{k}^{i}}{\sum_{j=1} \tilde{\omega}_{k}^{j}}
$$

\subsection{Selection of Proposal Distributions}

The selection of a suitable form of importance function to represent the true posterior density is a crucial step in the particle filter ((Arulampalam et al., 2002); (Rawlings and Bakshi, 2006)). The conventional approach is to use the state transition density as the proposal distribution/importance function, i.e. $q\left[\left(x_{k}^{i} \mid x_{k-1}^{i}\right), y_{k}\right] \approx P\left[x_{k}^{i} \mid x_{k-1}^{i}\right]$, and draw particles from the above importance function. Because the state transition function (being used as importance function) does not take in to account the most recent observation, $y_{k}$, the particles drawn from transition density may have very low likelihood, and their contributions to the posterior estimation become negligible. It may be noted that the use of appropriate importance function can significantly reduce the number of particles required for generating accurate estimates, as compared to the conventional particle filter (Arulampalam et al., 2002). In general, it is difficult to design such a proposal and the choice of proposal distribution is highly problem dependent.

The computational steps involved are as follows (Arulampalam et al., 2002):

\subsubsection{Initialization}

At $k=0, M$ samples are drawn from the given distribution of initial the state, $\hat{x}_{0 \mid 0}$.

\subsubsection{Importance Sampling}

At the $k^{\prime}$ th time step, after obtaining measurement $y_{k}, M$ observers (EKF or UKF or IEKF) are used in parallel to compute means and covariances of the proposal distributions, i.e. $\bar{x}_{k \mid k}^{i}, \bar{P}_{k \mid k}^{i}$ for each propagated particle $\hat{x}_{k-1 \mid k-1}^{i}$. The importance density is then approximated as $q\left[\left(x_{k}^{i} \mid x_{k-1}^{i}\right), y_{k}\right] \approx N\left[\bar{x}_{k \mid k}^{i}, \bar{P}_{k \mid k}^{i}\right]$ and used to draw a sample around each particle.

\subsubsection{Computation of Weights}

The weights associated with each particle are now computed by Eq. (9), and These $\tilde{\omega}_{k}^{i}$ weights are then normalized to obtain $\omega_{k}^{i}$ as given by Eq. (10).

\subsubsection{Re-sampling}

This step involves discarding samples that have low importance and reassigning weights to the remaining particles. Various approaches have been suggested in the literature for carrying out this step.

In our proposed algorithm we used the residual resampling algorithm.

\section{HYBRID ITERATED KALMAN PARTICLE FILTER}

Before talking about our proposed algorithm (Hybrid Iterated Kalman Particle Filter), firstly the unscented Kalman filter and the iterated extended kalman filter are introduced.

\subsection{Unscented Kalman Filter}

The Unscented Kalman Filter belongs to a bigger class of filters called Sigma-Point Kalman Filters or Linear Regression Kalman Filters, which are using the statistical linearization technique ((Gelb, 1974); (Julier, 2002); (Julier et al., 2002);(Julier and Uhlmann, 2004); (Lefebvre and Bruyninckx, 2004)) . This technique is used to linearize a nonlinear function of a random variable through a linear regression between $\mathrm{n}$ points drawn from the prior distribution of the random variable. The UKF is founded on the intuition that it is easier to approximate a probability distribution that it is to approximate an arbitrary nonlinear function or transformation (Julier and Uhlmann, 2004). The sigma points are chosen so that their mean and covariance to be exactly $x_{k-1}^{a}$ and $P_{k-1}$. Each sigma point is then propagated through the nonlinearity yielding in the end a cloud of transformed points. The new estimated mean and covariance are then computed based on their statistics. This process is called unscented transformation. The unscented transformation is a method for calculating the statistics of a random variable which undergoes a nonlinear transformation (Wan and van der Merwe, 2001).

\subsection{Iterated Extended Kalman Filter}

The extended Kalman filter (EKF) is a minimum mean-square-error (MMSE) estimator based on the Taylor series expansions of the nonlinear functions $f(\cdot)$ and $h(\cdot)$ around the current estimates.

In the EKF, the state distribution is represented by using a Gaussian random variable. It only uses the linear expansion terms. 
Model and Observation:

$$
\begin{aligned}
& x_{k}=f\left(x_{k-1}\right)+v_{k-1} \\
& y_{k}=h\left(y_{k}+u_{k}\right)
\end{aligned}
$$

Initialization:

$$
x_{0}^{a}=\mu_{0} \text { with error covariance } P_{0}
$$

Model Forecast Step/Predictor:

$$
\begin{gathered}
x_{k}^{f} \approx f\left(x_{k-1}^{a}\right) \\
P_{k}^{f}=J_{f}\left(x_{k-1}^{a}\right) P_{k-1} J_{f}^{T}\left(x_{k-1}^{a}\right)+Q_{k-1}
\end{gathered}
$$

Data Assimilation Step/Corrector:

$$
\begin{gathered}
x_{k}^{a} \approx x_{k}^{f}+K_{k}\left(y_{k}-h\left(x_{k}^{a}\right)\right) \\
K_{k}=P_{k}^{f} J_{k}^{T}\left(\hat{x}_{k}\right)\left(J_{h}\left(x_{k, i}^{a}\right) P_{k}^{f} J_{k}^{T}\left(x_{k}^{a}\right)+R_{k}\right)^{-1} \\
P_{k}=\left(I-K_{k} J_{h}\left(x_{k}^{a}\right)\right) P_{k}^{f}
\end{gathered}
$$

In the EKF, $h(\Delta)$ is linearized about the predicted state estimate $x_{k}^{f}$. The IEKF (Liang-qun et al., 2005) tries to linearize it about the most recent estimate, improving this way the accuracy ((Lefebvre and Bruyninckx, 2004); (Gelb, 1974) ). This is achieved by calculating $x_{k}^{a}, K_{k}, P_{k}$ at each iteration. Denote $x_{k, i}^{a}$ the estimate at time $k$ and $i$ th iteration. The iteration process is initialized with $x_{k, 0}^{a}=x_{k}^{f}$. Then the measurement update step becomes for each $i$ :

$$
\begin{gathered}
x_{k, i}^{a} \approx x_{k}^{f}+K_{k}\left(y_{k}-h\left(x_{k, i}^{a}\right)\right) \\
K_{k, i}=P_{k}^{f} J_{k}^{T}\left(\hat{x}_{k, i}\right)\left(J_{h}\left(x_{k, i}^{a}\right) P_{k}^{f} J_{k}^{T}\left(x_{k, i}^{a}\right)+R_{k}\right)^{-1} \\
P_{k, i}=\left(I-K_{k, i} J_{h}\left(x_{k, i}^{a}\right)\right) P_{k}^{f}
\end{gathered}
$$

\subsection{Hybrid Iterated Kalman Particle Filter}

Our proposed algorithm named hybrid iterated Kalman particle filter is a combination of the UKF and the IEKF. The HIKPF inherits the excellent properties of the UKF and IEKF and can make efficient use of the latest observations, which make it very attractive for the generation of proposal distribution within the particle filtering framework.

At time $k$, the UKF is firstly used to update the particles, and to obtain the state estimate $\tilde{x}_{k, u f}$, and the corresponding covariance estimate $P_{k, u f}^{i}$, then the particles are updated using the IEKF with $\bar{x}^{k, u f}$, and
$P_{k, u f}^{i}$. After the IEKF-update, the final state and covariance estimates $\bar{x}_{k}^{i}$ and $\hat{P}_{k_{j}}^{i}$ of time step $k$ are obtained.

Using the estimates, the required proposal distribution $N\left(\bar{x}_{k_{j}}^{i}, \hat{P}_{k_{j}}^{i}\right)$ is formed. Here, samples can be drawn from the approximated distribution $N\left(\bar{x}_{k_{j}}^{i}, \hat{P}_{k_{j}}^{i}\right)$.

The following figure shows the flow chart of our proposed algorithm.

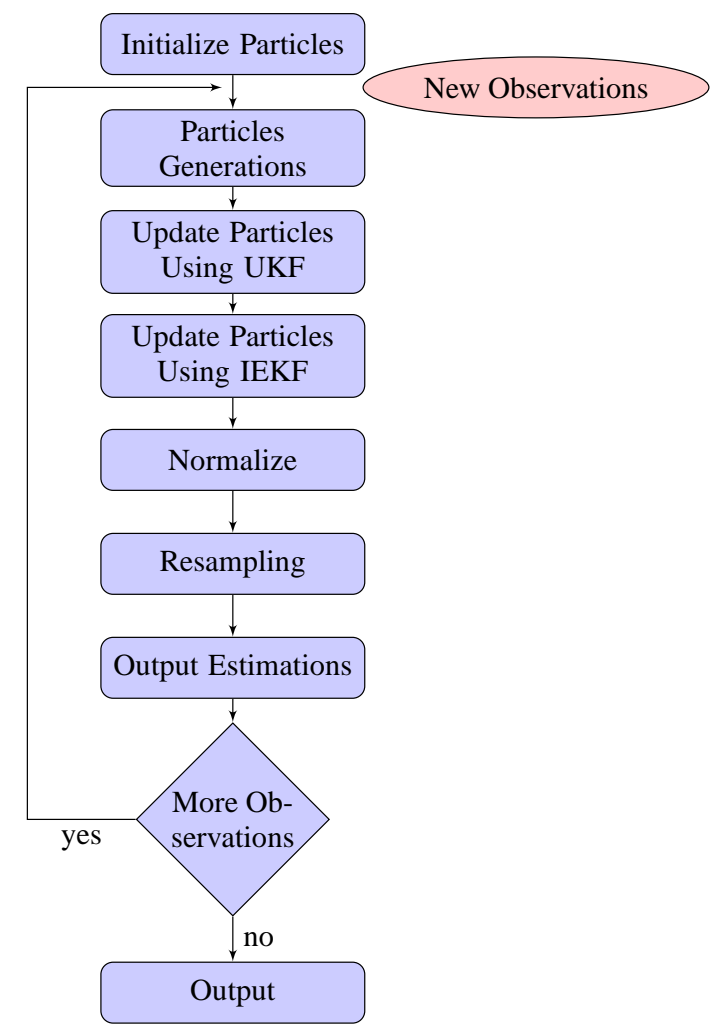

Figure 1: The schematic description of the proposed algrithm (HIKPF).

\section{HIKPF Algorithm Steps}

Step 1.Initialization: $k=0$

FOR $i=1, \ldots ., N_{p}$

Draw the particles $x_{0}^{i}$ from the prior $P\left(x_{0}\right)$ and set:

$\bar{x}_{0}^{i}=E\left(x_{0}^{i}\right)$

$P_{0}^{i}=E\left[\left(x_{0}^{i}-\bar{x}_{0}^{i}\right)\left(x_{0}^{i}-\bar{x}_{0}^{i}\right)^{T}\right]$

$\bar{x}_{0, a}^{i}=E\left[\left(x_{0, a}^{i}\right)\right]=\left[\left(x_{0}^{i}\right)^{T}, 0,0\right]^{T}$

$P_{0, a}^{i}=E\left[\left(x_{0, a}^{i}-\bar{x}_{0, a}^{i}\right)\left(x_{0, a}^{i}-\bar{x}_{0, a}^{i}\right)^{T}\right]=\operatorname{diag}\left(P_{0}^{i} Q R\right)$

END FOR

Step 2. FOR $k=1,2, \ldots$

(1)FOR $i=1, \ldots \ldots, N_{p}$ 
(a) Update the particles using the UKF Calculate the sigma points

$$
\chi_{k-1, a}^{i}=\left[\bar{x}_{k-1, a}^{i} \bar{x}_{k-1, a}^{i} \pm \sqrt{\left(n_{a}+\lambda\right) P_{k-1, a}^{i}}\right]
$$

Propagate samples into future and compute the one-step-ahead estimates:

$$
\begin{aligned}
& \chi_{k \mid k-1, x}^{i}=f\left(\chi_{k-1, x}^{i}, \chi_{k-1, v}^{i}\right) \\
& Y_{k \mid k-1, u f}^{i}=h\left(\chi_{k \mid k-1, x}^{i}, \chi_{k-1, u}^{i}\right) \\
& \bar{x}_{k \mid k-1, u f}^{i}=\sum_{j=0}^{2 n_{a}} W_{m}^{(j)} \chi_{k \mid k-1, x}^{(j) i} \\
& P_{k \mid k-1, u f}^{i}=\sum_{j=0}^{2 n_{a}} W_{c}^{(j)}\left[\chi_{k \mid k-1, x}^{(j) i}-\bar{x}_{k \mid k-1, u f}^{i}\right]\left[\chi_{k \mid k-1, x}^{(j) i}-\right. \\
&\left.\bar{x}_{k \mid k-1, u f}^{i}\right]^{T}
\end{aligned}
$$

$\bar{y}_{k \mid k-1, u f}^{i}=\sum_{j=0}^{2 n_{a}} W_{m}^{(j)} Y_{k \mid k-1, u f}^{(j) i}$

Incorporate the new observation $y_{k}$, and update the one-step-ahead estimates to obtain $\bar{x}_{k, u f}^{i}$

$$
\begin{gathered}
P_{y_{k} y_{k}}=\sum_{j=0}^{2 n_{a}} W_{c}^{(j)}\left[Y_{k \mid k-1, u f}^{(j) i}-\bar{y}_{k \mid k-1, u f}^{i}\right]\left[Y_{k \mid k-1, u f}^{(j) i}-\bar{y}_{k \mid k-1, u f}^{i}\right]^{T} \\
P_{x_{k} y_{k}}=\sum_{j=0}^{2 n_{a}} W_{c}^{(j)}\left[\chi_{(j) i_{k \mid k-1}}-\bar{x}_{k \mid k-1, u f}^{i}\right]\left[Y_{k \mid k-1, u f}^{(j) i}-\bar{y}_{k \mid k-1, u f}^{i}\right]^{T} \\
K_{k, u f}=P_{x_{k} y_{k}} P_{y_{k} y_{k}}^{-1} \\
\bar{x}_{k, u f}^{i}=\bar{x}_{k \mid k-1, u f}^{i}+K_{k, u f}\left(y_{k}-\bar{y}_{k \mid k-1, u f}^{i}\right) \\
P_{k, u f}^{i}=P_{k \mid k-1, u f}^{i}-K_{k, u f} P_{y_{k} y_{k}} K_{k, u f}^{T}
\end{gathered}
$$

(b) Use the IEKF to update estimations obtained through UKF update process

FOR $i=1, \ldots ., N_{p}$

Compute the Jacobians $F_{k}^{i} \& G_{k}^{i}$ of the process model

Update the particles with the IEKF

$$
\begin{aligned}
\bar{x}_{k \mid k-1, i e f}^{i} & =f\left(\bar{x}_{k, u f}^{i}\right) \\
P_{k \mid k-1, i e f}^{i} & =F_{k}^{i} P_{k, u f}^{i}\left(F_{k}^{i}\right)^{T}+G_{k}^{i}
\end{aligned}
$$

FOR $j=1: c$ ( $c$ is the number of iteration)

Compute the Jacobians $H_{k_{j}}^{i} \& U_{k_{j}}^{i}$ of the measurement model

Update the covariance and the state estimate from the following equations obtained from IEKF respectively .

$$
\begin{gathered}
K_{k_{j}, i e f}=P_{k_{j} \mid k_{j}-1, \text { ief }}^{i}\left(H_{k_{j}}^{i}\right)\left[U_{k_{j}}^{i} R_{k_{j}}\left(U_{k_{j}}^{i}\right)^{T}+\right. \\
\left.H_{k_{j}}^{i} P_{k_{j} \mid k_{j}-1, i e f}^{i}\left(H_{k_{j}}^{i}\right)^{T}\right]^{-1} \\
P_{k_{j}, \text { ief }}^{i}=P_{k_{j} \mid k_{j}-1, \text { ief }}^{i}-K_{k_{j}, \text { ief }} H_{k_{j}}^{i} P_{k_{j} \mid k_{j}-1, i e f}^{i} \\
\bar{x}_{k_{j}, i e f}^{i}=\bar{x}_{k_{j} \mid k_{j}-1, i e f}^{i}+K_{k_{j}, i e f}\left(y_{k_{j}}-h\left(\bar{x}_{k_{j}, i e f}^{i}\right)\right) \\
\text { let } \bar{x}_{k_{j}}^{i}=\bar{x}_{k_{j}, i e f}^{i}, \hat{P}_{k_{j}}^{i}=P_{k_{j}, \text { ief }}^{i}
\end{gathered}
$$

\section{END FOR}

$$
\text { Draw } x_{k}^{i} \sim q\left(x_{k}^{i} \mid x_{k-1}^{i}, z_{k}\right)=N\left(\bar{x}_{k_{j}}^{i}, \hat{P}_{k_{j}}^{i}\right)
$$

Assign the particle a weight, $w_{k}^{i}$, according to the equation below obtained from PF

\section{END FOR}

$$
w_{k}^{i} \propto w_{k-1}^{i} \frac{P\left(z_{k} \mid x_{k}^{i}\right) P\left(x_{k}^{i} \mid P\left(x_{k-1}^{i}\right)\right.}{q\left(x_{k}^{i} \mid x_{k-1}^{i}, z_{k}\right)}
$$

(2) Normalize the weights

$$
\begin{aligned}
\text { FOR } i=1, \ldots, N \\
w_{k}^{i}=\frac{w_{k}^{i}}{\sum_{j=1: N} w_{k}^{i}} .
\end{aligned}
$$

\section{END FOR.}

(3) Resample

(4) Output: calculate the required estimations using the particle set.

END FOR.

Step 3. $k=k+1$, go to Step2 or end the algorithm

\section{SIMULATION AND EXPERIMENTAL RESULTS}

The simulation results of the HIKPF algorithm is presented and discussed in this section as well as the comparison between HIKPF and the previously proposed algorithms including PF, PF-EKF, PF-UPF and PF-IEKF . The system models were taken from (R Van der Merwe, 2000) as following.

$$
\begin{gathered}
x_{k}=1+\sin (0.4 \pi k)+0.5 x_{k-1}+v_{k-1} \\
y_{k}= \begin{cases}0.2 x_{k}^{2}+e_{k}, & \text { if } k \leq 30 \\
0.5 x_{k}-2+e_{k}, & \text { if } k>30\end{cases}
\end{gathered}
$$

Where $v_{k}$ is a Gamma $\varsigma_{a}(3,2)$ random variable modelling the process noise, and the measurement noise $u_{k}$ is drawn from a Gaussian distribution $N(0,0.00001)$. In this experiment 200 particles are used and the program is repeated 100 times for timesteps $k=1, . .60$. The unscented transformation parameters are set to be $\alpha=1, \beta=0$, and $\kappa=2$. The 
output of the algorithm is the mean of samples set that can be computed $\hat{x}=\frac{1}{N} \sum_{j=1}^{N_{s}} x_{t}^{j}$. The mean square errors of each run is defined as

$$
M S E=\left(\frac{1}{T} \sum_{k=1}^{T}\left(\hat{x}_{k}-x_{k}\right)\right)^{\frac{1}{2}}
$$

Figure 2 Shows the true and the estimated state of the system HIKPF and the other methods. It is clear from the figure that particle filter (PF) and extended kalman particle filter ( PF-EKF) deviate from the true states at some time steps. The unscented Kalman particle filter(PF-UKF) and iterated extended kalman particle filter (PF-IEKF) gives better performance than $\mathrm{PF}$ and PF-EKF but less than our proposed system (HIKPF).

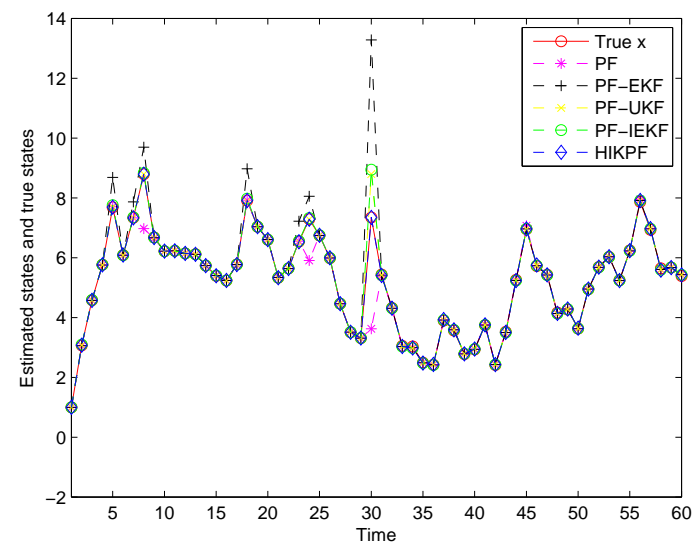

Figure 2: Estimation of the system state generated by different particles filters.

The performance evaluation of our system compared with other methods is shown in table 1. In this table, our proposed system (HIKPF) gives the best performance with the lowest mean and variance with mean value 0.015607 and variance value (Var) 0.00000395 .

Table 1: Estimation of means and variances of MSE of different particles filters over 100 independent runs.

\begin{tabular}{|c|c|c|}
\hline \multirow{2}{*}{ Algorithm } & \multicolumn{2}{|c|}{ MSE } \\
& mean & Var \\
\hline PF & 0.25881 & 0.057151 \\
\hline PF-EKF & 0.32392 & 0.021656 \\
\hline PF-UKF & 0.077684 & 0.006589 \\
\hline PF-IEKF & 0.049368 & 0.0015238 \\
\hline HIKPF & 0.015607 & 0.00000395 \\
\hline
\end{tabular}

Estimation of mean squars errors (MSEs) of different particle filters are shown in Figure 3 In this figure, it is clear that the bottom real line (Blue Line) is the HIKPF performance line. The proposed algorithm

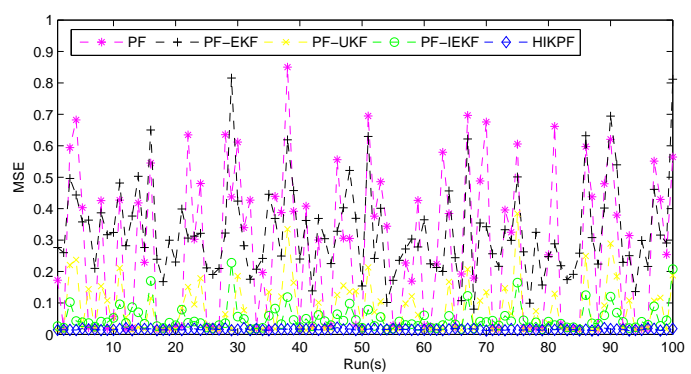

Figure 3: MSEs estimation of different particles filters at each run.

give the lowest mean square error at every independent run.

\section{CONCLUSIONS}

A new algorithm referred to as the hybrid iterated Kalman particle filter (HIKPF) is proposed. The proposed algorithm is developed from unscented Kalman filter (UKF) and iterated extended Kalman filter (IEKF) to generate the proposal distribution leading to efficient use of the latest observations and generates more close approximation of the posterior probability density. Numerical simulation and experiment results show that HIKPF algorithm is much robust than the previously proposed algorithms such as (PF, PF-EKF, PF-UPF and PF-IEKF).

\section{REFERENCES}

Arulampalam, M., Maskell, S., Gordon, N., and Clapp, T. (2002). A tutorial on particle filters for online nonlinear/non-gaussian bayesian tracking. In IEEE Transactions on Signal Processing. IEEE Signal Processing Society,Vol. 50, pp. 174-188.

Broida, T. and Chellappa, R. (1986). Estimation of object motion parameters from noisy images. In IEEE Transactions on Pattern Analysis and Machine Intelligence. IEEE Computer Society Washington, DC, USA,Vol. 8, No. 1, pp. 90-99.

Collins, R., Lipton, A., Kanade, T., Fujiyoshi, H., Duggins, D., Tsin, Y., Tolliver, D., Enomoto, N., and Hasegawa, O. (2000). System for video surveillance and monitoring. In Technical Report CMU-RI-TR-00-12. Robitics institute.

Fen, X. and Ming., G. (2010). Pedestrian tracking using particle filter algorithm. In International Conference on Electrical and Control Engineering. IEEE Computer Society Washington, DC, USA,pp.1478-1481.

Gelb, A. (1974). Applied Optimal Estimation. M.I.T. Press, Cambridge, 1st edition.

Gordon, N., Salmond, D., and Smith, A. (1993). Novel approach to nonlinear/non-gaussian bayesian state es- 
timation. In IEEF Proceedings Radar and Signal Processing. IET,Vol. 1, pp. 107-113.

Isard, M. and Blake, A. (1998). Condensation conditional density propagation for visual tracking. In International Journal of Computer Vision. Kluwer Academic Publishers Hingham,MA,USA, Vol. 29, No. 1, pp. 528.

Julier, S. (2002). The scaled unscented transformation. In Proceedings of the 2002 American Control Conference2. IEEE Conference Publications, Vol 6,PP.45554559.

Julier, S., Jeffrey, J., and Uhlmann, K. (2002). Reduced sigma point filters for the propagation of means and covariances through nonlinear transformations. In In Proceedings of the American Control Conference. IEEE Conference Publications Vol 2, pp. 887-892.

Julier, S. J. and Uhlmann, J. K. (2004). Unscented filtering and nonlinear estimation. In Proceedings of the IEEE. IEEE, Vol 92, pp. 401-422.

kyung-min Cho, jeong-hun Jang, and ki-sang Hong (2001). Adaptive skin-color filter. In Pattern Recognition. Elsevier, Vol 34,pp. 1067-1073.

Lefebvre, T. and Bruyninckx, H. (2004). Kalman filters for nonlinear systems: A comparison of performance. In International Journal of Control. Vol 77,pp.639-653.

Liang-qun, L., Hong-bing, J., and Jun-hui, L. (2005). The iterated extended kalman particle filter. In Proceedings International Symposium on Communication and Information Technologies 2005. IEEE Conference Publications, Vol 2,pp.1213-1216.

Lipton, A., Fujiyoshi, H., and Patil, R. (1998). Moving target classification and tracking from real-time video. In Proceeding of IEEE Workshop Applications of Computer Vision. IEEE Conference Publicationsn,pp. 814.

LIU, Y., Haizho, A., and Guangyou, X. (2001). Moving object detection and tracking based on background subtraction. In Proceeding of Society of Photo-Optical Instrument Engineers. Vol. 4554, pp. 62-66.

McIvor, A. M. (2000). Background subtraction techniques. In Proceeding of Image and Vision Computing. IVCNZ00, Hamilton, New Zealand.

Meyer, D., Denzler, J., and Niemann, H. (1998). Model based extraction of articulated objects in image sequences for gait analysis. In Proceeding of IEEE Int. Conf. Image Proccessing. IEEE Conference Publications, Vol 3,pp.78-81.

Phung, S., Chai, D., and Bouzerdoum, A. (2003). Adaptive skin segmentation in color images. In Proceeding of IEEE International Conference on Acoustics, Speech and Signal Processing. IEEE Conference Publications, Vol. 3, pp. 353-356.

R Van der Merwe, A. D. (2000). The unscented particle filter. In Advances in Neural Information Processing Systems.

Rawlings, J. and Bakshi, B. (2006). Particle filtering and moving horizon estimation. In Computers and Chemical Engineering. Elsevier, Vol 30,pp. 1529-1541.

Ristic, B., Arulampalam, S., and Gordon, N. (2004). Beyond the Kalman filter: Particle filters for tracking applications. Artech House.
Soderstorm, T. (2002). Discrete-time stochastic systems, in: Advanced Textbooks in Control and Signal Processing. Springer.

Sugandi, B., Kim, H., Tan, J. K., and Ishikawa, S. (2009). A moving object tracking based on color information employing a particle filter algorithm. In Artificial Life and Robotics. Springer Japan, Vol 14,pp. 39-42.

Wan, E. and van der Merwe, R. (2001). Chapter 7: The unscented kalman filter,. In Kalman Filtering and Neural Networks, S. Haykin, Ed.,. Wiley Publishing.

Zhiqiang, W., Peng, Z., Deng, X., and Li.Shifeng (2011). Particle filter object tracking based on multiple cues fusion. In Advanced in Control Engineering and Information Science. Procedia Engineering,Vol 15, pp. 1461-1465.

Zhonga, Q., Qingqinga, Z., and Tengfeia, G. (2012). Moving object tracking based on codebook and particle filter. In International Workshop on Information and Electronics Engineering. Procedia Engineering,Vol 29,pp. 174-178. 